\title{
[gw22-e0790] EFFECTS OF CHRONIC INTRAUTERINE HYPOXIC ON MYOCARDIAL INDUCIBLE NITRIC OXIDE SYNTHASE AND PLASMA ENDOTHELIN-1 IN OFFSPRING RATS
}

Zi-Yang Huang ${ }^{1}$, Hong-An Ying ${ }^{2}$, Zhen-Hua Wang ${ }^{11}$ The Second Affiliated Hospital Of Fujian Medical University, Fujian, China; ${ }^{2}$ The First Peoples Hospital Of Taizhou, Taizhou, China

\subsection{6/heartjnl-2011-300867.72}

Objective To study the effects of chronic intrauterine hypoxic on myocardial inducible nitric oxide synthase (iNOS) and plasma endothelin-1 (ET-1).

Methods Chronic intrauterine hypoxic rat was divided into three groups: a hypoxic group, an air mimic control group and a blank control group. Each group studied 10 offspring rats. We monitored the weight of organs and birth weight of offspring, immunohistochemistry was used to examined the expression of myocardial iNOS, enzyme linked immunosorbent assay (ELISA) measured the plasma ET-1 expression of offspring at one day and six-month old.

Results Organ/body weight percentage shows chronic intrauterine hypoxia can cause a disproportionate growth of offspring organs. Myocardial iNOS immunohistochemistry shows the expression of myocardial cells of the hypoxic group significantly increased compared with that of the air mimic control group and that of the blank control group $(p<0.05)$, but the air mimic control group was not significantly different from that of the blank control group ( $p>0.05)$. ELISA shows there was no significant difference of the expression of the plasma ET-1 of all groups of offspring at 1 day old, $p>0.05$. But it shows there was a significant difference of the expression of the plasma ET-1 of the hypoxic group compared with that of the air mimic control group and that of the blank control group of offspring at six-month old $(\mathrm{p}<0.05)$. At the same time, the air mimic control group was not significantly different from that of the blank control group ( $p>0.05)$.

Conclusions Intrauterine chromic hypoxia may be a stress factor for development of the cardiovascular system, it can induce low birth weight and high expression of myocardial iNOS and plasma ET-1, which leads to increase the risk of cardiovascular disease. 